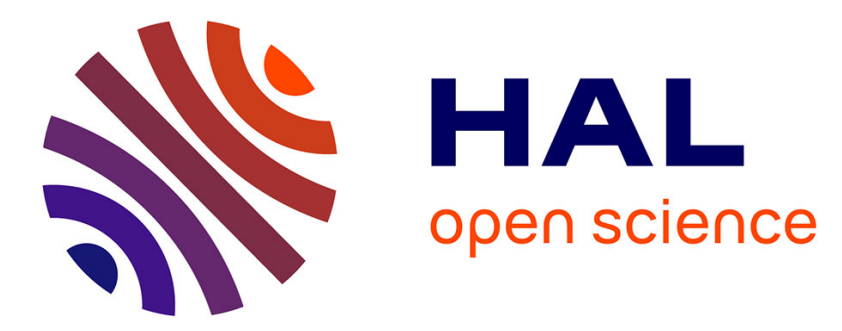

\title{
How does the dielectrophoresis affect the soot dendrite growth on resistive sensors?
}

Adrien Reynaud, Mickael Leblanc, van Bastian Ranarivelo, Stéphane Zinola, Philippe Breuil, Jean-Paul Viricelle

\section{- To cite this version:}

Adrien Reynaud, Mickael Leblanc, van Bastian Ranarivelo, Stéphane Zinola, Philippe Breuil, et al.. How does the dielectrophoresis affect the soot dendrite growth on resistive sensors?. Sensors and Actuators A: Physical , 2021, 327, pp.112729. 10.1016/j.sna.2021.112729 . emse-03230852

\section{HAL Id: emse-03230852}

\section{https://hal-emse.ccsd.cnrs.fr/emse-03230852}

Submitted on 1 Jun 2021

HAL is a multi-disciplinary open access archive for the deposit and dissemination of scientific research documents, whether they are published or not. The documents may come from teaching and research institutions in France or abroad, or from public or private research centers.
L'archive ouverte pluridisciplinaire HAL, est destinée au dépôt et à la diffusion de documents scientifiques de niveau recherche, publiés ou non, émanant des établissements d'enseignement et de recherche français ou étrangers, des laboratoires publics ou privés. 


\title{
How does the dielectrophoresis affect the soot dendrite growth on resistive sensors?
}

\author{
A. Reynaud ${ }^{1,2}$, V. B. Ranarivelo ${ }^{1}$, M. Leblanc ${ }^{1}$, S. Zinola ${ }^{1}$, P. Breuil ${ }^{2}$, and J.-P. \\ Viricelle ${ }^{2}$ \\ ${ }^{1}$ IFP Energies nouvelles, Rond-point de l'échangeur de Solaize, BP 3, 69360 Solaize, France ; Institut Carnot \\ IFPEN Transports Energie \\ ${ }^{2}$ Mines Saint-Étienne, Univ Lyon, CNRS, UMR 5307 LGF, Centre SPIN, 42100 Saint-Étienne, France
}

\begin{abstract}
The European standards "Euro" limit the emission of several pollutants from thermal engine vehicles. The particle number $(\mathrm{PN})$ and particulate mass (PM) emissions are specifically limited since 2011, hence modern Diesel engines are equipped with a Diesel particulate filter (DPF). Onboard diagnostic (OBD) regulations impose to perform the self-diagnostic of the DPF. Nowadays, this diagnostic is done through a very simple backpressure monitoring, but the forthcoming steps will probably require a more sophisticated system, such as a resistive soot sensor. The aim of this study is to investigate the mechanisms leading to the formation of bridge-like soot deposits brought to light by scanning electron microscopy. A 2D model taking into account the aerodynamic transport and the electrostatic effects was used to improve our understanding of the soot deposition process. The model presented in this paper includes a force that has been neglected so far in previous works about resistive soot sensors: the dielectrophoresis. In this theoretical frame, this force affects particles motion in such a way that they tend to numerically build the bridge-like structures that are observed experimentally. Simulations showed that the dielectrophoresis mainly impacts large particles - over $150 \mathrm{~nm}$ diameter.
\end{abstract}

\section{Introduction}

Internal combustion engines are producing several kinds of pollutants including soot particle aerosols. The typical size of the soot particles ranges from $10 \mathrm{~nm}$ to $1000 \mathrm{~nm}$ with a median diameter around $100 \mathrm{~nm}$. Those data may significantly vary according to the engine, the combustion process and many other parameters $[1,2]$. The small size of the particles make them able to deeply penetrate through the breathing apparatus. Moreover, several carcinogenic compounds [3] are adsorbed at the particle surface during the combustion process or the cooling process involved by the exhaust phase. Thus, the inhalation of such aerosols has harmful consequences including breathing diseases or cancers [4, 5, 6]. Consequently, several organizations are regulating those emissions through standards.

In order to respect the limitations set by the European Union, a common strategy is to add a particulate filter to the exhaust line of thermal engine vehicles. However, the efficiency of the filter may vary over time. In order to detect potential filter failures, the on-board diagnostic is performed using sensors. Currently, the loading state of the filter is evaluated by measuring the pressure drop but this technique does not allow to detect small leaks, which can lead to a significant increase of

E-mails: M. Leblanc (mickael.leblanc@ifpen.fr), S. Zinola (stephane.zinola@ifpen.fr), P. Breuil (pbreuil@emse.fr), J.-P. Viricelle (viricelle@emse.fr)

ORCIDs: A. Reynaud: 0000-0003-0684-9286, M. Leblanc: 0000-0002-5661-5798, J.-P. Viricelle: 0000-0002-7293-6591

DOI: $10.1016 /$ j.sna.2021.112729 


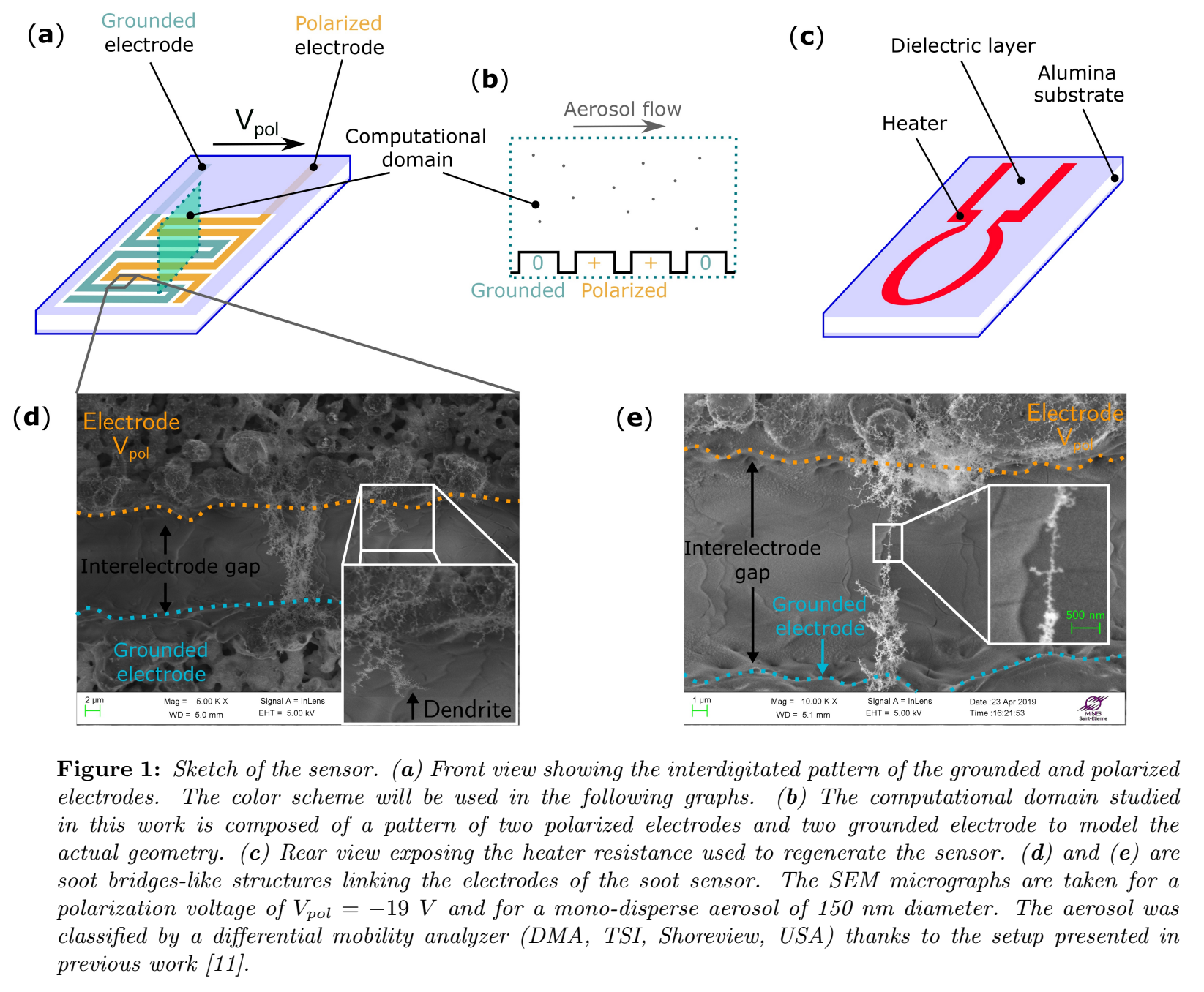

the tailpipe emissions. Many new technologies are currently under developpement to perform more accurate measurements. Such technologies include the radio-frequency sensors [7], the electric charge sensors $[8,9]$ or the accumulative sensors. The latter include the capacitive and resistive sensors [10].

In this work, we will be focused on the resistive sensor technology [12]. Its working principle is based on the measurement of the conductivity of the soot particles. It is composed of a polarized electrode and a grounded electrode that are manufactured by depositing the platinum ink on an alumina substrate by screen printing and laser engravement. The geometry of such electrodes can be interdigitated to optimize the length of the inter-electrode gap for a given surface of the sensitive element. Indeed, when the soot particles are deposited between the electrode, they build electrically conductive bridge-like structures (Figure 1). The signal transmitted by the sensor is the intensity of the current crossing the soot structures. Grondin et al. show that the deposition of the particles on the electrodes is increased by electrostatic phenomena: the higher the polarisation tension, the lower the response time of the sensor [13]. It was concluded that the polarisation tension may affect the soot bridge building velocity. However, this behavior is limited by the Joule effect: up to a certain point, the electrical current burns the bridges. Thus, Grondin et al. find out an optimum polarization voltage to balance the construction and destruction phenomenon. This point is highly dependent on the soot chemistry. Furthermore, the influence of the thermophoresis has been studied to evaluate its positive or negative impact on the collection efficiency $[13,14,15]$. The particle mass flow around the 
sensor $[12,16]$, the aerodynamics in the vicinity of the sensor $[17,18]$ are experimental parameters studied to improve the technological development of resistive sensors.

However, only a little number of studies deal with numerical and theoretical analysis of the sensor to describe the physics underlying the soot deposition on the electrodes of a resistive sensor. Firstly, the work of Teike et al. should be mentionned to study the building of the soot structures at the microscale [19]. Their model is based on the lagrangian tracking of soot particles in the direct vicinity of the electrodes of a resistive soot sensor. The particles are submitted to the brownian motion, the electrophoresis (Coulomb force) and the drag force. The main feature of their model is the electric and aerodynamic coupling with the particle deposition. Indeed, according to the Teike et al. model, the soot structures influence both the electrostatic field and the aerodynamic field which are reponsible of the typical shapes of the bridge-like structures. In addition to the Teike et al. model, the work of Fragkiadoulakis et al. [20] brings another level of understanding by modeling the sensor response based on the collection efficiency computed for several physical phenomena. They include the convective diffusion, the thermophoresis, the inertial impaction, the turbulent impaction and the electrophoresis. Those two research works deal with the simulation of the resistive sensor system from two different points of view and they both consider the action of the electric field on the particles through one phenomenon: the electrophoresis.

However, another force may apply to a particle immersed into an electric field: the dielectrophoretic force (DEP). This force applies to conductive particles moving in an inhomogeneous electric field [21]. Due to their conductivity, the particles are polarized and as the electric field is inhomogeneous, the poles experience opposed Coulomb forces with different magnitudes leading to a migration. Dielectrophoresis is widely used for biological application because it helps manipulating cells in liquid medium. It is also interesting to manipulate other kind of conductive particles including polymere, metal, viruses, DNA molecules or proteins according to [22]. Dielectrophoresis was also demonstrated to be useful to collect airborne particles for applications such as electret filters [23, 24] or micro-electric precipitators [25]. Another use of the dielectrophoresis is presented by Wasisto et al. [26] who designed a micro-cantilever type sensor using dielectrophoresis to increase the collection efficiency.

The target of this study is to investigate the influence of the particle size on the dendrite construction by means of a numerical approach. First, the trajectories of soot particles are computed, then the dendrite construction are simulated. The work presented in the present paper is based on the Teike et al. model and the dielectrophoretic force was added to the force equilibrium. Also, another electrode geometry was investigated.

\section{Methodology}

Two objectives should be distinguished. The first one is to compute particle trajectories in the vicinity of the electrodes. To do so, the motion equation has to be solved. The dendrites locally modify the electric field. To take it into account, Teike et al. suggest to couple the soot deposit with the aerodynamic and electrostatic fields computation [19]. This method is illustrated by Figure 2. The next paragraphs are describing the numerical method step by step.

\subsection{Step 1: initialization of the fields}

In order to compute the drag and the Coulomb force acting on each particle, and thus to compute the motion equation, the calculation of aerodynamic and electric fields is required. Notice that this step is repeated everytime a collision occurs, but with a modified geometry.

\subsubsection{Aerodynamic field}

Lattice-Boltzmann method (LBM) The aerodynamic field was computed numerically by solving the Boltzmann equation which describes the space and time fluctuations of the velocity distribution 


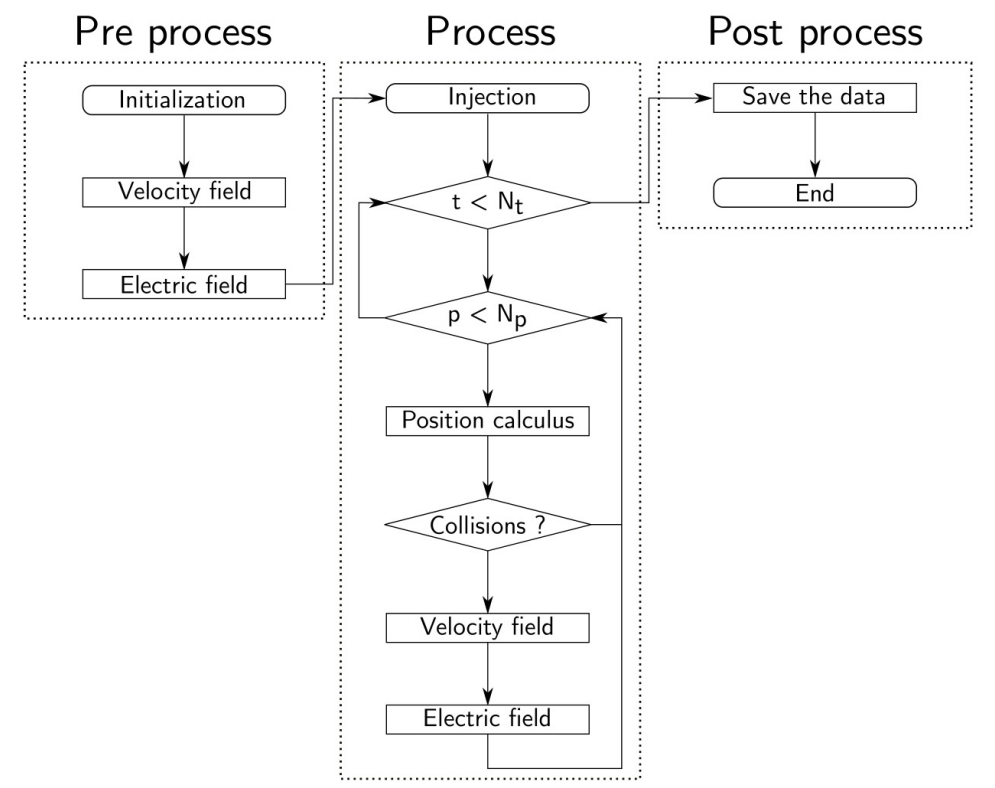

Figure 2: Overview of the methodology. The velocity field is computed with the lattice-Boltzmann method, the electric field is calculated with a finite element method and the position is updated using the Verlet algorithm. The general algorithm is based on the work of Teike et al. [19] and has been adapted to this work.

of the molecules in the fluid, called microscopic velocity $\vec{\xi}$. With a single relaxation time approach:

$$
\frac{\partial f}{\partial t}+\vec{\xi} \cdot \vec{\nabla} f=\frac{1}{\tau}\left(f-f^{e q}\right)
$$

where $\tau$ is the relaxation time to reach the equilibrium distribution function $f^{e q}$. From the microscopic velocity, the density and the momentum given by:

$$
\rho=\int f d \vec{\xi} \text { and } \rho \vec{v}=\int \vec{\xi} f d \vec{\xi}
$$

In order to solve (1), it has to be discretised in space, time, and microscopic velocity dimensions. The D2Q9 model is a numerical scheme that allows to write the lattice Boltzmann equation (LBE):

$$
f_{i}\left(\vec{x}+\vec{\xi}_{i} \Delta t, t+\Delta t\right)-f_{i}(\vec{x}, t)=-\frac{\Delta t}{\tau}\left(f_{i}(\vec{x}, t)-f_{i}^{e q}(\vec{x}, t)\right)
$$

where $\Delta t$ is the time step. The equilibrium distribution function is:

$$
f_{i}^{e q}=\rho w_{i}\left(1+\frac{\vec{e}_{i} \cdot \vec{u}}{c_{s}^{2}}+\frac{\left(\vec{e}_{i} \cdot \vec{u}\right)^{2}}{2 c_{s}^{4}}-\frac{u^{2}}{2 c_{s}^{2}}\right)
$$

where $c_{s}=\frac{1}{\sqrt{3} \frac{\Delta x}{\Delta t}}, w_{i}$ are a weigthing coefficients and $e_{i}$ are the discrete velocities (cf. Figure 3$)$. The D2Q9 scheme gives:

$$
\vec{e}_{i}= \begin{cases}(0 ; 0) & \text { if } i=0 \\ (1 ; 0),(0 ; 1),(-1 ; 0),(0 ;-1) & \text { if } i=\{1 ; 2 ; 3 ; 4\} \\ (1 ; 1),(-1 ; 1),(-1 ;-1),(-1 ; 1) & \text { if } i=\{5 ; 6 ; 7 ; 8\}\end{cases}
$$

and:

$$
w_{i}= \begin{cases}4 / 9 & \text { if } i=0 \\ 1 / 9 & \text { if } i=\{1 ; 2 ; 3 ; 4\} \\ 1 / 36 & \text { if } i=\{5 ; 6 ; 7 ; 8\}\end{cases}
$$




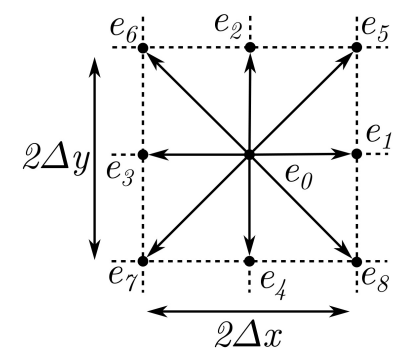

Figure 3: Discretised velocities of the D2Q9 scheme.

Finally, the macroscopic properties of the fluid are given by:

$$
\rho=\sum_{i=0}^{8} f_{i} \text { and } \rho \vec{v}=\sum_{i=0}^{8} \vec{\xi}_{i} f_{i}
$$

Boundary conditions The aim of this study is to investigate the particle kinematic at the electrodes scale, hence the flow above the electrodes is simulated within the short range of $150 \mu \mathrm{m}$ above the electrodes (cf. Figure 4). It was modelled by a Couette flow which was translated, in terms of boundary condition, into a shear flow of the top boundary. The fixed velocity condition was implemented using the Zou and He [27] method. The intensity of the velocity of the top boundary was computed by a 3D simulation of the flow around the whole sensor in the experimental conditions described in a previous work [11]. It was founded that the velocity was between $u_{t o p}=0.05 \mathrm{~m} / \mathrm{s}$ and $u_{\text {top }}=0.2 \mathrm{~m} / \mathrm{s}$ depending on the location on the sensitive element. Consequently, this range of velocity magnitude will be investigated in the following. The calculations leading to those results are reported in the $\mathrm{PhD}$ thesis of Reynaud [28].

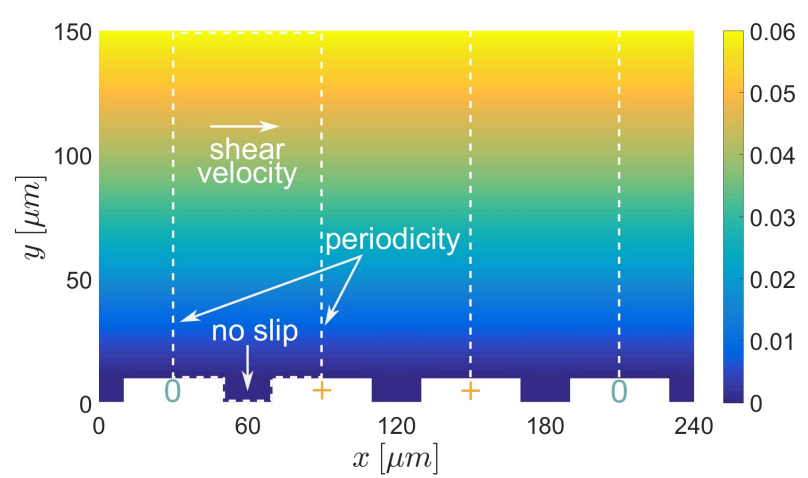

(a) $u_{x}$ in $m . s^{-1}$

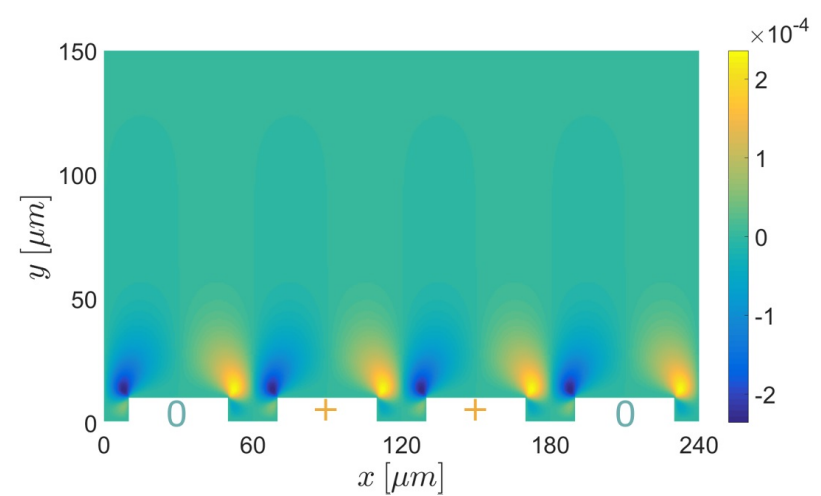

(b) $u_{y}$ in $m . s^{-1}$

Figure 4: Flow field in the vicinity of the electrodes $\left(\Delta x=500 \mathrm{~nm}, u_{\text {top }}=0.06 \mathrm{~m} . \mathrm{s}^{-1}\right)$. The flow field is computed on one fraction of the domain (dashed lines) and duplicated to extend it to the whole computational domain.

The electrodes were modelled as walls with no-slip condition. Finally, to simplify and to light the calculations, the lateral boundary conditions were defined as periodic boundaries. The sensitive element of the soot sensor was considered as large enough $(5000 \mu \mathrm{m})$ to be modeled by an infinite plate at the scale of an elementary pattern $(60 \mu \mathrm{m})$. Hence, the converged solution was duplicated several times to generate the whole computational domain where the particles were moving. 


\subsubsection{Electric field}

To compute the 2D electric field, the software FEMM (Finite Element Method Magnetics), developed by D. Meeker [29], was used. It supports a convenient control in Matlab through command lines and was adapted to the evolution of the geometry of the electrodes. This software uses the finite element method to solve numerically the Poisson equation:

$$
\triangle V=-\frac{\rho_{c}}{\varepsilon_{0}}
$$

where $V$ is the electric potential, $\rho_{c}$ the charge density and $\varepsilon_{0}$ the vaccuum permittivity. In our application, the charge density can be neglected, thus the right hand side of the Poisson equation is zero. The electric field $\vec{E}$ is derivated from the potential:

$$
\vec{E}=-\vec{\nabla} V
$$

To model an infinite height of air above the sensor, the voltage was fixed to $V_{\infty}=0 \mathrm{~V}$ at the height $y=1000 \mu \mathrm{m}$. Experimentally, half of the electrodes were polarized by a constant voltage $V_{\text {pol }}$ within the range $10-200 \mathrm{~V}$. The other electrodes were grounded. As they are printed on alumina, it is assumed that the substrate is a nonconducting material. Hence, the boundary condition for the gap between the electrodes was set as a zero-flux condition. Finally, the hypothesis was made that the sensor may be considered as an infinite plane. Thus, periodic boundary conditions are applied to the lateral sides of the computational domain, as shown in Figure 5 (a). As well as the electric field itself, the gradient of the squared norm was computed. It is indeed required to calculate the dielectrophoretic force.

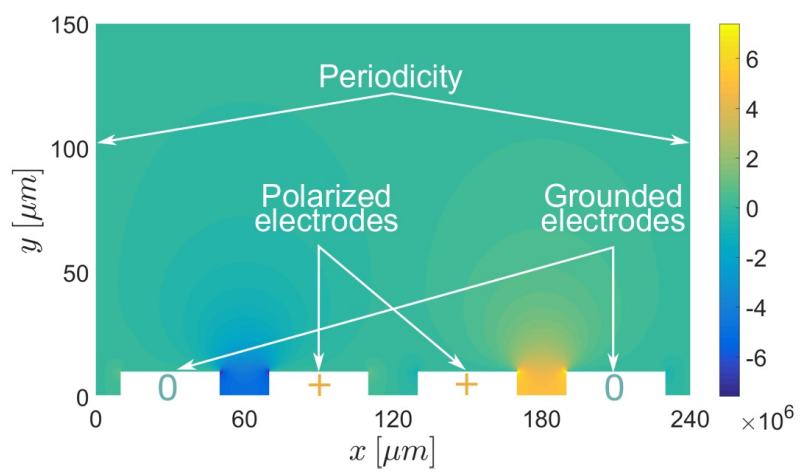

(a) $E_{x}$ in $V \cdot m^{-1}$

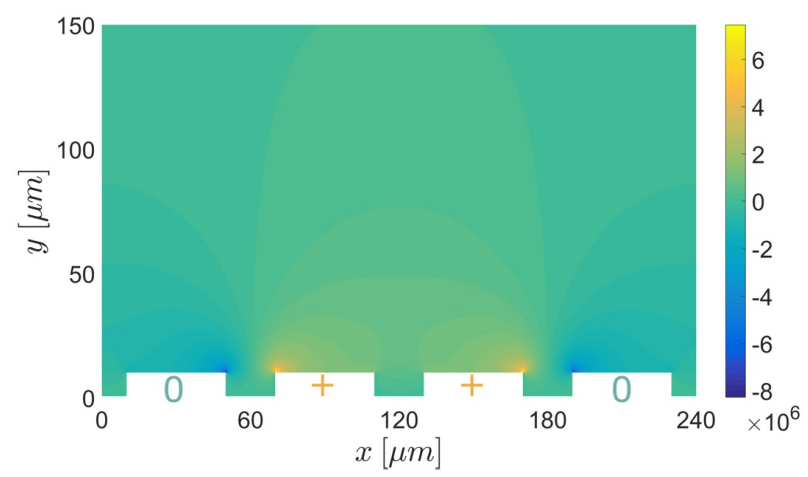

(b) $E_{y}$ in $V \cdot m^{-1}$

Figure 5: $X$ and $Y$ components of the electric field in the vicinity of the electrodes $\left(V_{\text {pol }}=20 \mathrm{~V}\right)$.

\subsection{Step 2: motion equation}

The objective of this model is to simulate nanoparticles in suspension in a gaseous medium and immersed within an electric field. The motion equation is the keystone of this approach and the trajectories of one soot particle is derivated from an ordinary differential equation (ODE).

The motion equation takes into account four phenomena: the brownian motion, the viscous effect of the gas, the electrophoresis and the dielectrophoresis. Due to their small mass, the gravitational effects were neglected. It is written as:

$$
\begin{cases}\frac{d \vec{x}}{d t} & =\vec{v} \\ m \frac{d \vec{v}}{d t} & =\sum \vec{F}_{e x t}\end{cases}
$$


where $\vec{x}$ is the position of the particle, $\vec{v}$ is its velocity, $m$ its mass, and $\sum F_{\text {ext }}$ refers to all the forces acting on the particle. The Verlet algorithm was chosen to solve (10). It is specifically dedicated to problems in which position, velocity and acceleration are involved, such as the motion equation. The numerical scheme is [30]:

$$
\left\{\begin{array}{l}
\vec{x}_{n+1}=\vec{x}_{n}+\Delta t \times \vec{v}_{n}+\frac{\Delta t^{2}}{2} \times \vec{a}_{n} \\
\vec{v}_{n+1}=\vec{v}_{n}+\frac{\Delta t}{2} \times\left(\vec{a}_{n}+\vec{a}_{n+1}\right)
\end{array}\right.
$$

where $\Delta t$ is the time step. Every forces acting on soot particle in the vicinity of the sensor electrodes are examined in this section.

Langevin force The first phenomenon that acts inherently on a nanoparticle immersed in fluid is the brownian motion. To model it, the Langevin force is used by many authors [19, 31].

$$
\vec{F}_{L}(t)=\vec{\zeta} \sqrt{\frac{216 \nu_{f} k_{b} T_{f}}{\pi \rho_{f} d_{p}^{5}\left(\frac{\rho_{p}}{\rho_{f}}\right)^{2} C_{c}\left(d_{p}\right) \Delta t}}
$$

where $k_{b}$ the Boltzmann constant and $\nu_{f}, T_{f}, \rho_{f}$ are the fluid dynamic viscosity, temperature and density. $d_{p}$ and $\rho_{p}$ are the diameter and the density of the particle and $\vec{\zeta}$ is a zero-mean, unit-varianceindependent Gaussian random number. $\Delta t$ is the time step of the numerical scheme of the "brownian dynamics" method, proposed by [32]. $C_{c}\left(d_{p}\right)$ is the Cunningham slip correction factor. It is used to correct the no-slip condition and is significant for high Knudsen numbers $\left(K n=2 \lambda / d_{p}\right)$, where $\lambda$ is the molecules mean-free path. It is given by the relation [33]:

$$
C_{c}(K n)=1+K n\left(\alpha+\beta e^{-\frac{\gamma}{K n}}\right)
$$

where $\alpha=1.142, \beta=0.558$ et $\gamma=0.999$ are empiric constants for solid particles.

Drag force The viscous effects are taken into account by the drag force. The Stokes' law can be used to compute the drag acting on spherical nanoparticles because the Reynolds number is usually lower than unity [34]:

$$
\vec{F}_{d r a g}=\chi \frac{3 \pi \eta_{f} d_{p}}{C_{c}\left(d_{p}\right)} \overrightarrow{v_{r}}
$$

where $\eta_{f}$ is the kinematic viscosity of the fluid, $\chi$ is the dynamic shape factor used to take into account the fractal morphology of the aggregates [35]. The relative velocity $\overrightarrow{v_{r}}$ is the difference between the fluid and the particle velocities.

Coulomb force The electrophoresis, that is the migration of charged particles under the influence of an electric field, is modelled by the Coulomb force given by:

$$
\overrightarrow{F_{E}}=n_{p} e \vec{E}
$$

where $n_{p}$ is the number of elementary charges $e$ carried by the particle. Indeed, it is assumed that combustion aerosol are slightly electrically charged. The charge number carried by any particle follows the Boltzmann equilibrium distribution [36].

Dielectrophoretic force A conductive particle immersed into an electric field is polarized. If the electric field is inhomogeneous, the electrostatic force magnitude and direction acting on every poles of the particle are different. From the unbalance of the electrostatic forces results a torque that rotate the particle and line the poles up to the field lines. Moreover, the particle migrates and might be either attracted or repelled by the zone of high electric density depending on the electric properties of both 
the particle and the medium it is immersed in. For a spherical particle immersed in a steady electric field, the analytical expression of the force is [22, 21]:

$$
\vec{F}^{D E P}=2 \pi \varepsilon_{f} r_{p}^{3}\left(\frac{\sigma_{p}-\sigma_{f}}{\sigma_{p}+2 \sigma_{f}}\right) \vec{\nabla} E^{2}
$$

where $\sigma_{f}$ is the conductivity of the medium, $\sigma_{p}$ is the conductivity of the particle and $r_{p}$ its radius. In the case of airborne soot particles $\sigma_{p} \gg \sigma_{f}$, leading to the approximation $\frac{\sigma_{p}-\sigma_{f}}{\sigma_{p}+2 \sigma_{f}} \approx 1$. Consequently, the sense of the vector is given by the sign of the term $\vec{\nabla} E^{2}$.

\subsection{Step 3: collision}

Collisions may occur between particles and electrodes or particles and dendrites. In order to detect collisions, a simple collision mesh was implemented as shown in Figure 6. The collision mesh cells might be either the same size $\Delta x_{\text {coll }}$ than the electric and aerodynamic fields' mesh cells size or bigger as reported in the Table 1. For the values used in this study, the size of the cells is not expected to influence significantly the dendrite morphology, as reported by Teike et al. [19] for $0.5 \mu m, 1 \mu m$ and $2 \mu m$.

Table 1: Values of the mesh parameters.

\begin{tabular}{llll}
\hline$d_{p}$ & $K_{\text {dep }}$ & $\Delta x$ & $\Delta x_{\text {coll }}$ \\
{$[\mathrm{nm}]$} & {$[-]$} & {$[\mathrm{nm}]$} & {$[\mathrm{nm}]$} \\
\hline 100 & 5 & 500 & 500 \\
125 & 4 & 500 & 500 \\
150 & 3 & 500 & 500 \\
250 & 4 & 500 & 1000 \\
500 & 2 & 500 & 1000 \\
\hline
\end{tabular}

The collision test is performed between the squared elements and the round particles and it is considered positive when the geometrical shapes overlap. One collision counter was implemented for each cell of the mesh. Thus, whenever a collision occurs, the counter is incremented. When it reaches the threshold value, called $K_{d e p}$, according to the model of Teike et al. [19], the cell is no longer considered as gas, but is turned into solid as part of one of the electrodes. As the ability of the particles to fill the cells changes according to their diameter, the values of the threshold $K_{d e p}$ are

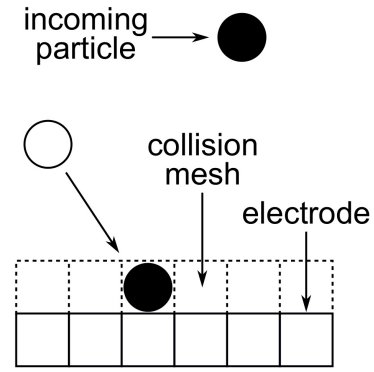

(a) $t_{1}$

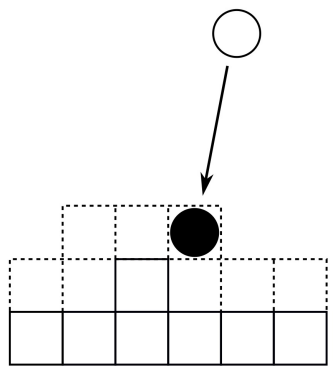

(b) $t_{2}$

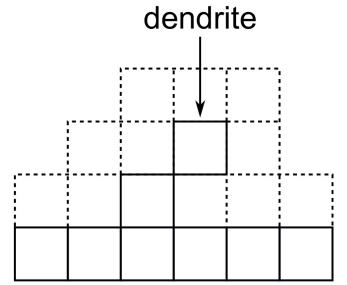

(c) $t_{3}$

Figure 6: Illustration of the collision process for an hypothetical case of $K_{\text {dep }}=1$. When the final position (black circle) of a particle is within a cell of the collision mesh (a), the electrode is extended to this cell (b). Dendrite growth by iterating this mechanism (c). 
reported in Table 1. It was assumed that the particles are stacked in the cells as chain-like structures, hence the particle number per cell is defined by $d_{p} / \Delta x_{\text {coll }}$.

It instantly inherits the boundary conditions of the electrodes they are captured by. Hence, for a particle deposited on the grounded electrode, the geometry of this electrode is extended, leading to an update of the electric field and the aerodynamic field, as described in Figure 2. Furthermore, the physical model in this work does not take into account elastic or inelastic collision. It is assumed that once particles are captured by the electrodes, they are never released.

\section{Results}

In this section will be exposed the results obtained by using the model described previously. First, the electric field will be analyzed to understand the phenomenology of the soot deposition. Then, the influence of the diameter on the particle deposition will be examined statistically thanks to the trajectory calculations. Finally, the dendrite growth will be studied to understand the mechanisms leading to this phenomenon.

\subsection{Electric forces}

\subsubsection{Phenomenology}

The magnitude of the electric field and the field lines, shown in Figure 7 (a), provide information about the Coulomb force. The electric field is represented for the specific case of $V_{p o l}=20 \mathrm{~V}$ in order to make the visualization easier. If the polarization voltage is higher, the zone $\mathrm{A}$ would have been less visible in the computational domain because an offset would have raised the limit between zones $\mathrm{A}$ and B. Figure 7 (a) shows that the positive particles are repelled in the zone A, but attracted in the zone $\mathrm{B}$ by the grounded electrodes. Regarding the negative particles, they are unconditionally attracted by the polarized electrodes. Naturally, this phenomenon is inverted if the sensor polarization sign is switched. Finally, the force vectors are oriented from an electrode surface to the other. No preferential deposition site, on an electrode surface, can be drawn from the analysis of the Coulomb force.

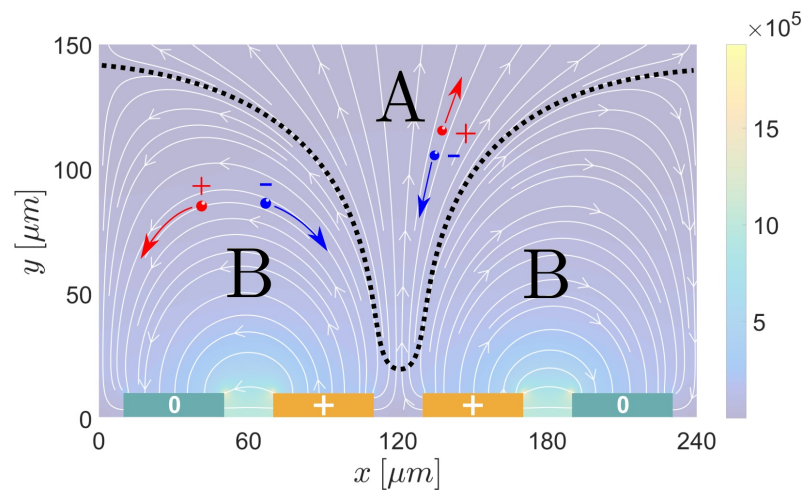

(a) $\|\vec{E}\|$ in $V \cdot m^{-1}$

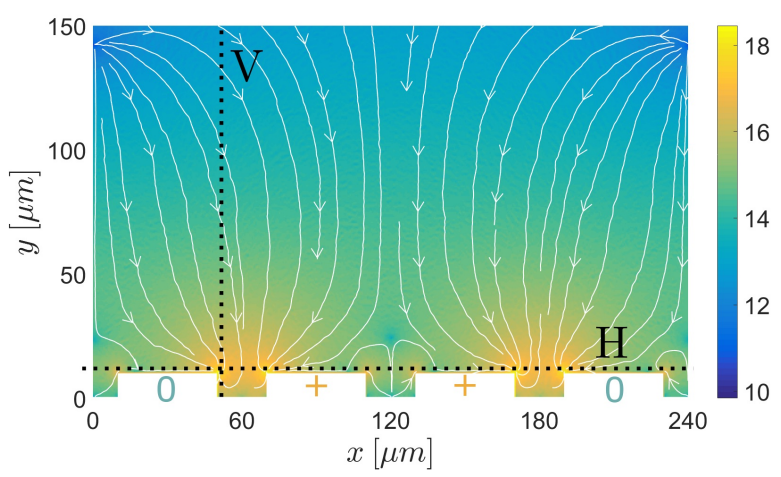

(b) $\log \left(\vec{\nabla} E^{2}\right)$

Figure 7: Electric field and gradient in the vicinity of the electrodes $\left(V_{\text {pol }}=20 \mathrm{~V}\right)$. (a) The Coulomb force attracts negative particles in the zones $A$ or $B$, but the positive particles are only attracted in the zone $B$. (b) The electric field gradient is represented in the computational domain. The electric forces (Coulomb force and dielectrophoretic force) are plotted in Figure 8 against the $H$ (horizontal) and $V$ (vertical) axis.

Moreover, the gradient of the electric field is shown in Figure 7 (b) and provides information about the dielectrophoretic force, because it is proportional to the term $\vec{\nabla} E^{2}$ according to the equation (16). The logarithm of $\vec{\nabla} E^{2}$ is plotted to improve the readability of this figure. It can be noticed that 
this term is strictly positive, meaning that the particles are always attracted by the zones of higher gradients. Such zones are the inter-electrode gaps insulating the polarized and the grounded electrodes. They also correspond to the zones where the bridge-like structures are detected by the sensor because the electric current may flow from one electrode to the other accross the soot particles.

To conclude, if we ignore the interaction with other forces, dielectrophoresis seems more likely than the Coulomb force to preferentially drive deposition to locations where signal-generating inter-electrode bridges can form.

\subsubsection{Quantitative analysis}

From the fields $\vec{E}$ and $\vec{\nabla} E^{2}$, it is easy to extract the magnitude of both the Coulomb force and dielectrophoretic force. In Figure 8, the forces are plotted against two axis: H and V (cf. Figure 7 (b)) to analyze their evolution along the two directions. The Coulomb force acting on particles of different diameters stays unchanged since it only depends on the electric charge. For this example, the electric charge was set to one elementary charge $e$. The dielectrophoretic force was computed for spheres of different diameters from $100 \mathrm{~nm}$ to $700 \mathrm{~nm}$. For all forces, the magnitude along the horizontal axis $\mathrm{H}$ increases in the vicinity of the inter-electrode gap. However, the increasing rate is higher for the dielectrophoretic force which increases by two order of magnitude. The Coulomb force only increases by one order of magnitude. By comparing the forces themselves, the dielectrophoretic force is higher than the Coulomb force if the sphere diameter is $300 \mathrm{~nm}$ or higher.

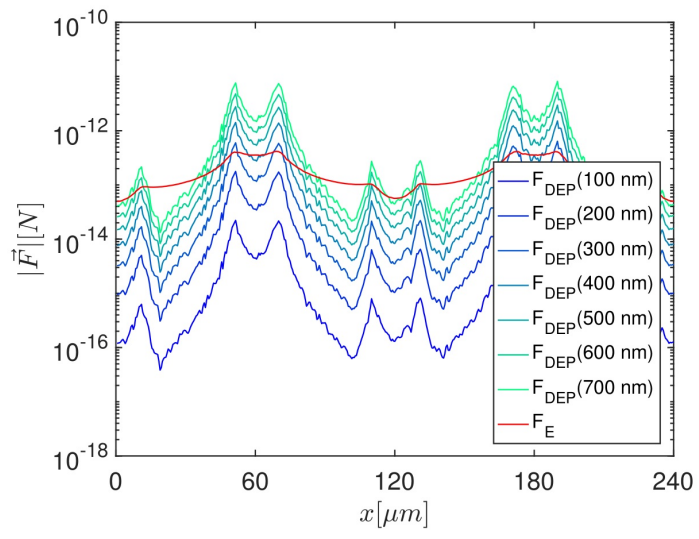

(a) Forces along axis $H$

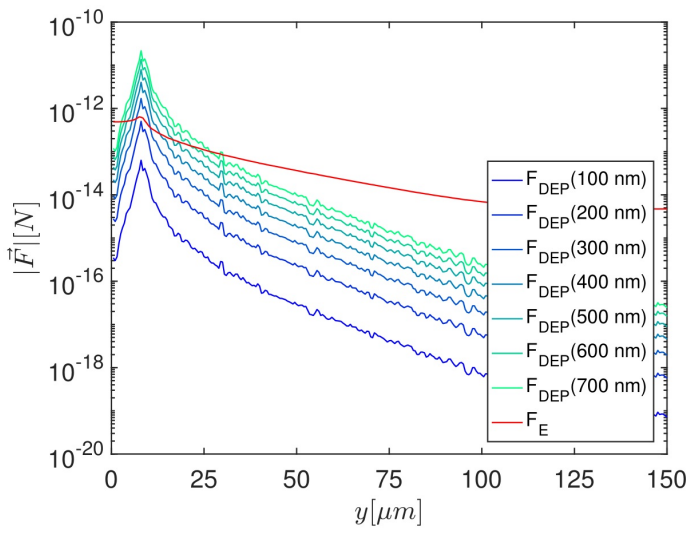

(b) Forces along axis $V$

Figure 8: Electric forces plotted along the $H$-axis and the $V$-axis for different particle diameters. The $x$ and $y$ coordinates correspond to the height and width of the computational domain.

The same conclusion can be drawn from the analysis of the forces along the vertical axis but it can be added that the dielectrophoretic effect decreases significantly faster than the intensity of the Coulomb force. Thus, the range of the Coulomb force is higher than the dielectrophoresis but for bigger particles, the dielectrophoresis drives the deposit at a short range.

\section{$3.2 \quad$ Kinematics}

The deposition process was first investigated by plotting the trajectories of the particles in the computational domain. The particle positions are initialized on the left of the domain, under the height of $100 \mu \mathrm{m}$ and the velocities are initialized by the local fluid velocity, computed by the LBM.

In Figure 9 (a), the trajectories of three $60 \mathrm{~nm}$ diameter particles and three $100 \mathrm{~nm}$ diameter particles are represented. The randomness of the trajectories is due to the brownian motion. The 


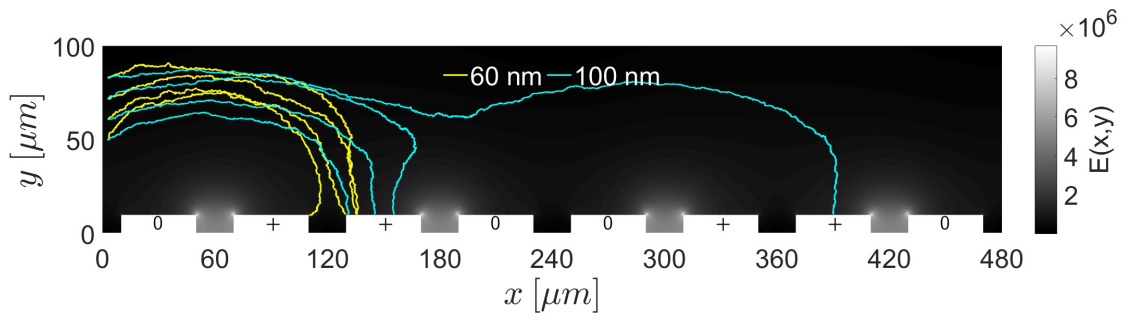

(a) $d_{p}=60 \mathrm{~nm}$ and $d_{p}=100 \mathrm{~nm}$

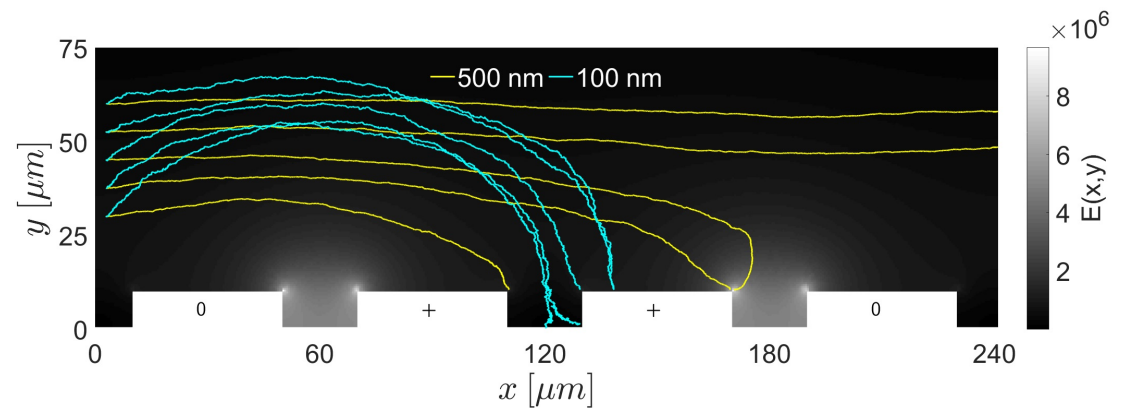

(b) $d_{p}=100 \mathrm{~nm}$ and $d_{p}=500 \mathrm{~nm}$

Figure 9: Trajectories of several particles of different diameters in the electric field and aerodynamic field of the vicinity of the sensor electrodes. The polarization voltage is $V_{\text {pol }}=60 \mathrm{~V}$, the shear velocity at $y=150 \mu \mathrm{m}$ is $u_{\text {top }}=0.06 \mathrm{~m} / \mathrm{s}$ and every particle is charged with a single (negative) elementary charge.

particles are all diffusing from the left to the right of the domain because of the direction of the shear flow. Since the flow velocity increases with the height above the electrodes, the drag force also increases. It also increases with the particle diameter as suggested by the equation (14). Unlike the drag force, the magnitude of both the Coulomb force and the dielectrophoresis decreases with the height above the electrodes. Thus, the ratio between the aerodynamic force and the electric forces increases with height. This explains why the particles injected at a higher y coordinate land later on the electrodes. This can be observed for the highest $100 \mathrm{~nm}$ diameter particle but it is not obvious for the smaller particles because they are less sensitive to the drag force. Indeed, the smallest particles closely follow the electric field force lines. According to those simulations, the smallest particles are not sensitive to the dielectrophoretic force, as suggested by Figure 8 .

Figure 9 (b) shows the trajectories of bigger particles. First, it can be noticed that the randomness of the trajectories is significantly lower for the $500 \mathrm{~nm}$ particles, which is consistent with the theory (cf. equation (12)). The behaviour of the particles toward the aerodynamic field is the same as in Figure 9 (a) which explains why the $500 \mathrm{~nm}$ particles are blown away and land slowly on the electrodes. The main difference between the $60 \mathrm{~nm}$ and the $500 \mathrm{~nm}$ particles is that the $500 \mathrm{~nm}$ particles tend to deposit preferentially on the angle of the electrodes, where the electric field gradient is the higher, as predicted by the calculations of Figure 7 (b).

\subsection{Deposit histogram}

The analysis of the particle trajectories provides information about the deposition phenomenology. It was learnt that the particle sensitive to the dielectrophoresis were attracted by the corners of the electrodes but the proportion of particles reaching those location was still unknown.

In order to understand where the particles land in a statistical point of view, 500 particles were released at the domain inlet and their deposition abscissa $x_{i}$ was analyzed. The initial particle height was computed so all of them are able to deposit on the first electrode pattern and not drifting to the 


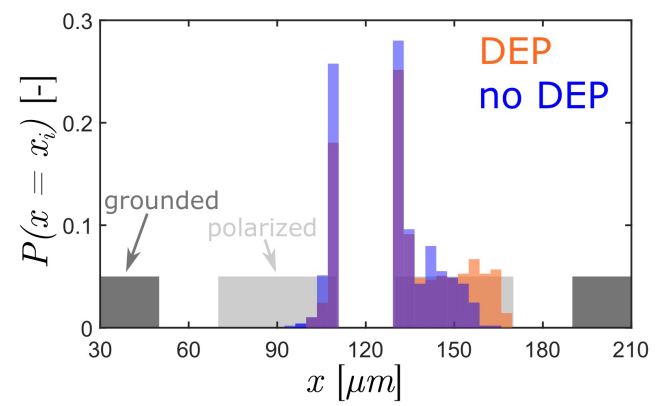

(a) $d_{p}=100 \mathrm{~nm}$

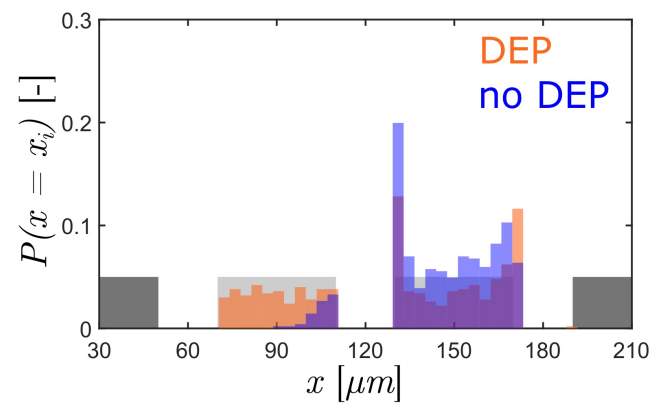

(c) $d_{p}=400 \mathrm{~nm}$

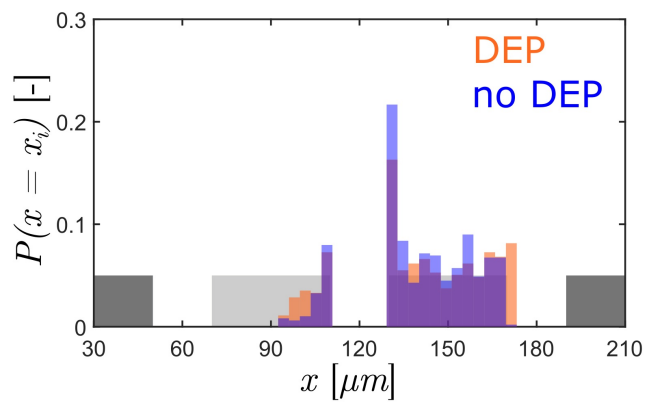

(b) $d_{p}=250 \mathrm{~nm}$

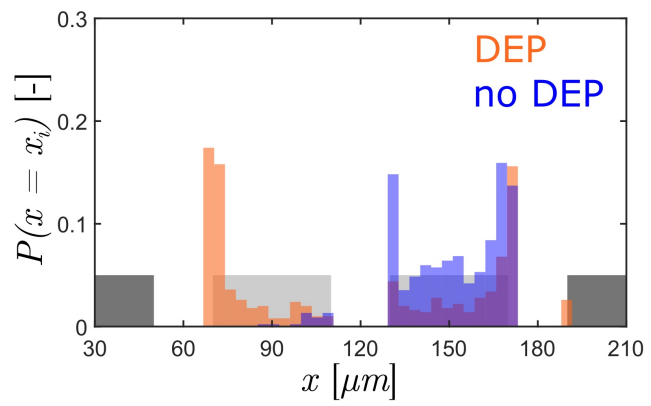

(d) $d_{p}=500 \mathrm{~nm}$

Figure 10: Deposit histograms for different particle diameters. The calculations have been performed with and without enabling DEP. The air flows from the left to the right of the horizontal axis. When superposed, the orange (with DEP) and blue (without DEP) histograms are purple.

second one as it can be see in Figure 9. Hence, the height was included between $30 \mu \mathrm{m}$ for $500 \mathrm{~nm}$ particle and $50 \mu \mathrm{m}$ for $100 \mathrm{~nm}$ particles. The histograms in Figure 10 sum up the probability particles have to drop off on a given abscissa. This probability is written $P\left(x=x_{i}\right)$. Further details about the methodology used to plot the deposition histogram is provided in [28].

Figure 10 shows the results of the calculations for four diameters from $100 \mathrm{~nm}$ to $500 \mathrm{~nm}$ with and without taking the dielectrophoresis into account. We do not report the results for lower diameters because the phenomenology is similar to the $100 \mathrm{~nm}$ particle behavior. First, it is important to highlight the general trend showed by the histograms: the higher the diameter, the higher the deposition rate around the inter-electrode gap between the polarized and the grounded electrodes. Two different phenomena can explain such a behaviour and they both depend on the particle diameter. For smaller particles $(100 \mathrm{~nm})$, the main force diffusing the particles from the left to the right is the drag. With an increasing diameter, the particles drop off more likely on the right side of the right polarized electrode because the drag magnitude increases. However for bigger particles the dielectrophoresis also has a significant impact on the particle kinematics (cf. Section 3.1). Since dielectrophoresis highly depends on the particle diameter (it is proportional to $d_{p}^{3}$ ), it is not suprising to observe that particles preferentially drop off close to the gap between the polarized and the grounded electrodes, as observed by analyzing the trajectories in the previous section. When the dielectrophoresis is enabled, the deposition rate in the inter-electrode regions is always higher regardless of the particle size (cf. Figure 10). Within the framework of this model, the Coulomb force tends to attract particles on the polarized side of the interelectrode gap. 


\subsection{Dendrite construction}

Finally, the system is simulated up to the dendrite construction. The aim of those simulations is to investigate which forces drive the dendrite construction process in order to provide a theoretical framework to model this phenomena. As explained by Figure 2, both the velocity field and the electric field are updated whenever a collision occures. Thus, the dendrite itself has an influence over its own building process because the electric field is modified by its growth. The electric field magnitude can be increased up to a factor ten, depending on the distance between the electrode and the dendrite tip. For this study, the particle are injected between the electrodes in the area defined by $x \in[50 ; 70] \mu m$ and $y \in[10 ; 20] \mu \mathrm{m}$ for the inter-electrode distance $d_{i e}=20 \mu \mathrm{m}$. When $d_{i e}=10 \mu \mathrm{m}$, the injection area is defined by $x \in[45 ; 55] \mu m$ and $y \in[10 ; 20] \mu m$. Twenty particles are randomly injected in this area every $10^{4}$ iterations. The total number of iterations being $10^{5}$, there is a total of 200 injected particles during the simulation.

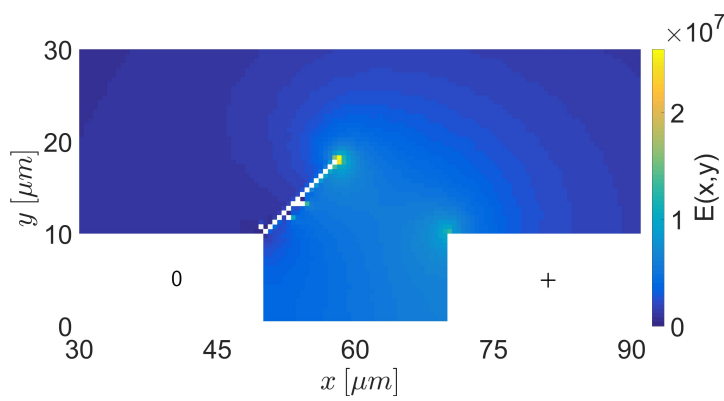

(a) No DEP, $d_{i e}=20 \mu m$

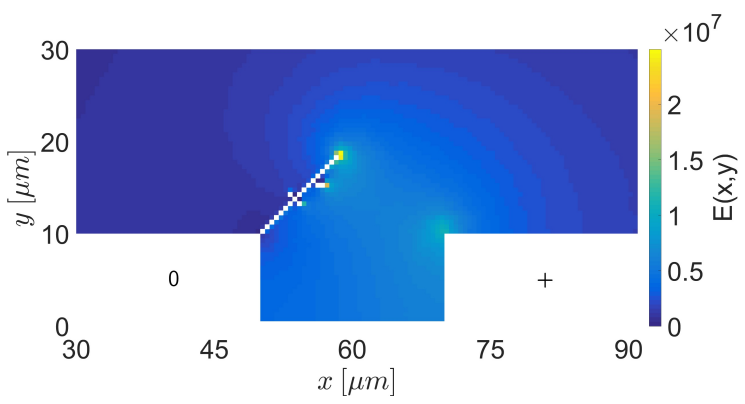

(c) $D E P, d_{i e}=20 \mu m$

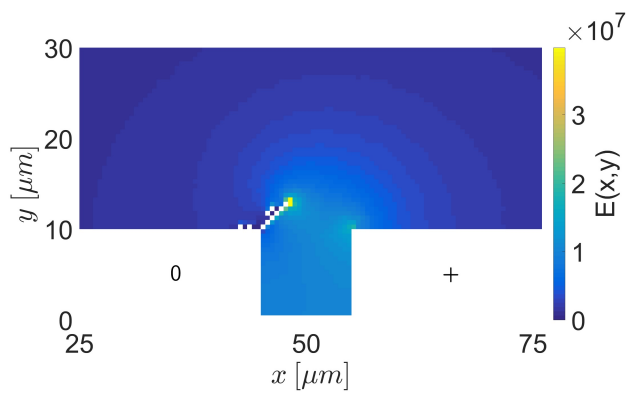

(b) No DEP, $d_{i e}=10 \mu \mathrm{m}$

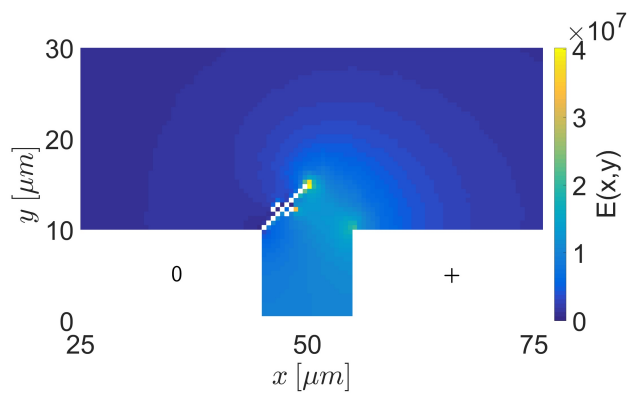

(d) $D E P, d_{i e}=10 \mu m$

Figure 11: Impact of the model and the inter-electrode gap distance $d_{i e}$ on the dendrite construction. Particles are singly positively charged, their diameter is $100 \mathrm{~nm}$. The polarization voltage is $V_{\text {pol }}=100 \mathrm{~V}$ and the shear velocity is $u_{\text {shear }}=0.2 \mathrm{~m} / \mathrm{s}$. The electric field magnitude unit is $\mathrm{V} / \mathrm{m}$.

Figure 11 presents the results obtained for $100 \mathrm{~nm}$ diameter particles and shows the influence of two parameters: the inter-electrode gap and the physical model. Indeed, in subfigures (a) and (b), the dielectrophoresis is not included in the force balance while it is in the other subfigures. In any case, the dendrites are straight, start from the grounded electrode and grow toward the polarized electrode. Indeed, the aerosol is only composed of positively charged particles and the dielectrophoresis effect is not strong enough to drag particles on the polarized electrode (as predicted in the section 3.1). The influence of the inter-electrode gap is minor and barely no difference can be observed comparing the two cases (a) and (c) for $d_{i e}=20 \mu \mathrm{m}$. However, the dendrite is slightly longer when the physical model includes the dielectrophoresis for $d_{i e}=10 \mu \mathrm{m}$. Indeed, reducing the inter-electrode gap leads to a higher dielectric force because it is proportionnal to the gradient $\vec{\nabla} E^{2}$. The DEP magnitude is higher in the context of a dendrite construction rather than the case of electrodes without any deposit, 


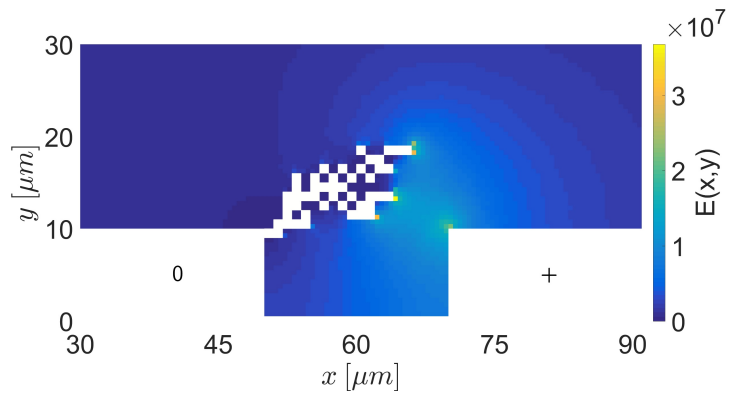

(a) No DEP, $V_{p o l}=100 \mathrm{~V}$

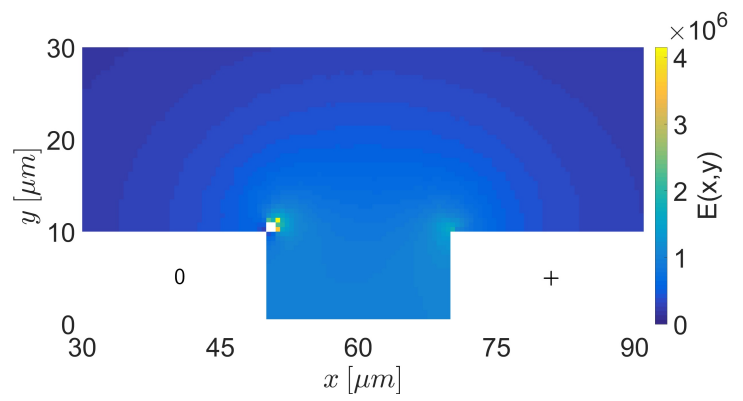

(c) $N o D E P, V_{\text {pol }}=20 \mathrm{~V}$

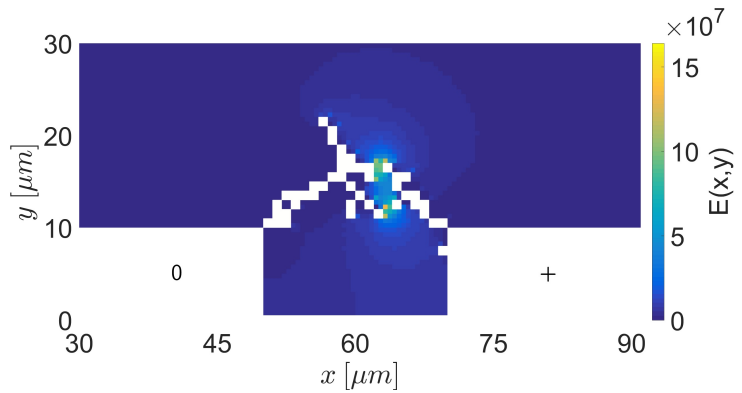

(b) $D E P, V_{p o l}=100 \mathrm{~V}$

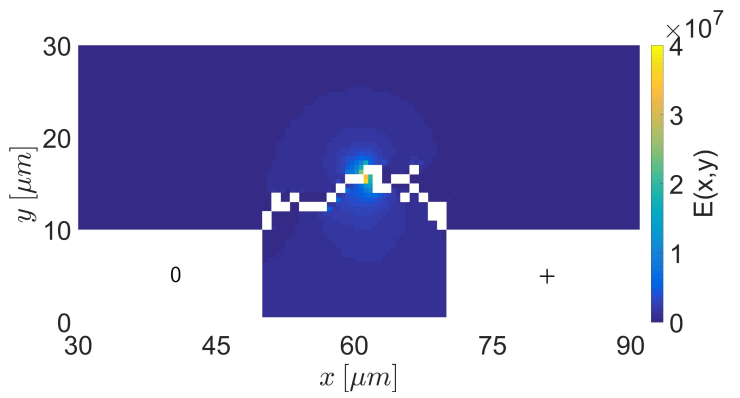

(d) $D E P, V_{\text {pol }}=20 \mathrm{~V}$

Figure 12: Impact of the model and of the polarization voltage on the dendrite construction. Particles are singly positively charged, their diameter is $500 \mathrm{~nm}$ and the shear velocity is $u_{\text {shear }}=0.2 \mathrm{~m} / \mathrm{s}$. The electric field magnitude unit is $\mathrm{V} / \mathrm{m}$. 
as considered previously in the section 3.1. As a conclusion, under the hypothesis of this model, the dielectrophoresis may affect the dendrite construction made of small airborne particles $(100 \mathrm{~nm})$ under specific conditions increasing the electric field gradient.

The same calculations were done for $500 \mathrm{~nm}$ diameter particles, as shown in Figure 12. At this point, it might be reminded that for such particles, the mesh parameters are $\Delta_{\text {coll }}=1000 \mu_{m}$ and $K_{d e p}=2$. It means that if only one particle lands on a cell, its state stays unchanged as a fluid cell. Two cases are studied in this work. In the first one, the DEP is not taken into account, hence the electric field acts on particle through the Coulomb force only. The results are shown on subfigures 12 (a) and (c). Even though the structure seems more chaotic than the results obtained for $100 \mathrm{~nm}$, the overall look is similar: the growth starts from the grounded electrode toward the polarized electrode but with no deposit on it. The particles deposit on the dendrite only because they are repelled from the polarized electrode by the Coulomb force. Finally, for a lower polarization voltage, the number of particles landing on the electrode is significantly smaller because they are blown away by the drag force and may not deposit until the end of the simulation. Hence, one might find it hard to consider that this structure could link the electrodes as observed experimentally.

When the dielectrophoresis is integrated to the force balance however, the dendritic growth phenomenology is deeply modified for bigger particles. Subfigures 12 (b) and (d) show indeed that the structure born from this physical model raises both from the polarized and the grounded electrodes. This building phenomenology leads to a structure which is more consistent with the experimental observation, that is to say bridge-like structures. In the mathematical point of view, the dielectrophoresis induces such results because the direction of the electric field itself has no influence on the force analytical expression. Indeed, the direction of the force is the one of the gradient of the square electric field $\vec{\nabla} E^{2}$. Thus, even if the Coulomb force repels positive particles from the polarized electrode, the attractive force of the dielectrophoresis acting on high diameter particles is higher.

However, this model was implemented with only two dimensions, which is a limitation for a realistic simulation of the dendrite growth. Indeed, when they are long enough to cover much of the interelectrode gap, it is likely that particles land on the dendrite branches before reaching the tip of the dendrite. This explains why the bridge of Figure 12 (b) is not complete.

\section{Experimental observations and discussions}

In previous studies on the same sensor $[13,37,11]$, tests were performed in a wide range of cases. In the latter study, the particle ability to build bridge-like stuctures was investigated for different electrical mobility diameters and different aerodynamic diameters. A propane diffusion flame burner (miniCAST, Jing Ltd, Switzerland) was in charge with the soot particle generation. The size classification process was performed by means of two devices: the DMA (Differential Mobility Analyzer, TSI, Shoreview, USA) and the AAC (Aerosol Aerodynamic Classifier, Cambustion, Cambridge, UK). The classified aerosol was then sent to the sensor and its signal was analyzed. More details on the experimental set-up can be founded in a previous paper [11].

The aerosol electrostatically classified by the DMA is composed of particles positively charged because of the physical principle of the DMA. The Scanning Electron Microscope (SEM) micrograph in Figure 13 shows a deposition pattern thanks to the contrast of each electrode. Light-colored electrodes correspond to a high soot deposit. The dark colour of the other electrodes means that a lower amount of soot particles are deposited on it. The polarization voltage applied to the sensor for this experiment was negative and equal to $V_{p o l}=-19 \mathrm{~V}$. Thus, the particles being positively charged, it can be reasonably assumed that they are preferentially attracted by the polarized electrodes due to the Coulomb force. Hence, the polarization of each electrode can be deduced from such micrograph. From this simple observation it can be concluded that for the monodisperse aerosol tested for this experiment $d_{m}=$ $150 \mathrm{~nm}$, the Coulomb force has a significant impact on the deposition mechanisms.

However, it was also noticed that small particles (mobility diameter between $60-150 \mathrm{~nm}$ and aerodynamic diameter between $50-70 \mathrm{~nm}$ ) had the ability to build bridge-like structures. They link electrodes together and it leads to a discrete conductivity step. The analysis of the times those events 


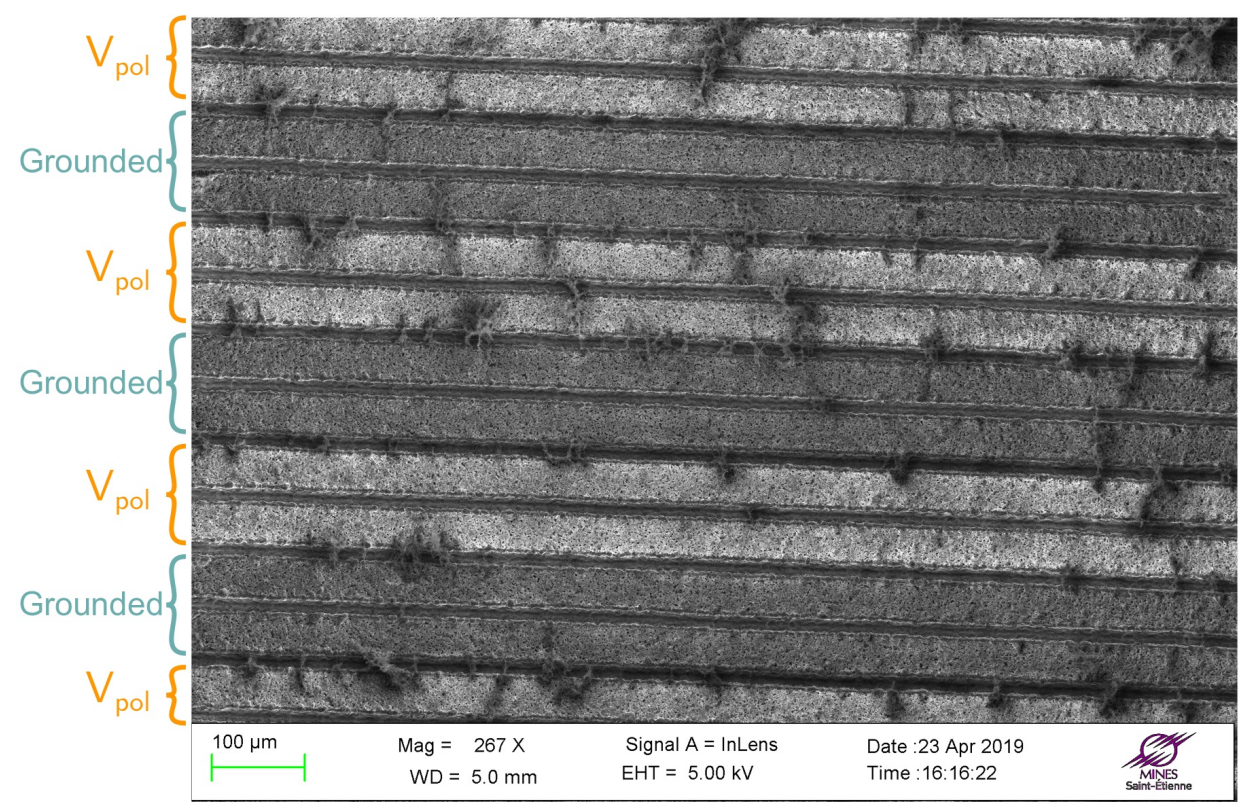

Figure 13: Monodisperse soot aerosol desposit of $d_{m}=150 \mathrm{~nm}$ diameter particles classified by means of a DMA [28]. The polarization voltage applied to the electrodes is $V_{\text {pol }}=-19 \mathrm{~V}$.

occur was performed, leading to the conclusion that smaller particle aerosols required more time (for a given concentration) to achieve the construction of the first soot bridge [11]. It was first interpretated as the impact of the geometric contribution of the particles: for the same flux of particles, the bridges get build faster by large particles because a lower number of them is required. Recently, those bridges were observed by means of a SEM as shown in Figure 1 [28]. They link the grounded and the polarized electrodes.

According to the model presented in this work, the dielectrophoresis plays an important role among the mechanisms leading to the soot bridge building. Indeed, it was shown that without DEP, the bridge cannot get closed because the Coulomb force repels the particles from the opposite electrode. The DEP however attracts the particles whatever their signs are on both sides of the interelectrode gap. Since actual bridges are observed, two hypothesis can be made:

- Hypothesis 1: the DEP also attracts the small particles at a short range (cf. Figure 14 (b)), which helps closing the bridges. The Coulomb force seems to be dominant at a long range (cf. Figure 13).

- Hypothesis 2: the dendrite collapses leading to the observed bridges (cf. Figure 14 (c)). Because the charges may be unequally distributed in the dendrite and the whole structure is immersed in an inhomogeneous electric field (cf. Figure 8), the DEP could be the responsible of the collapse.

To developp the hypothesis 1 , it cannot be ignored that according to the model, the DEP starts to affect significantly particles motion for $d_{p}=300 \mathrm{~nm}$. The model may have some imprecisions for several points that may impact the DEP force magnitude. For instance, the topology on the electrodes in the model is simplified by four rectangles while the real surface is more complex. Due to the platinum ink and the fabrication process of the sensor (laser engravement), the surface of the electrode is relatively high, especially in the regions of the inter-electrode gaps. This may change significantly the electric field gradient and consequently the DEP force magnitude. A 3D model would be useful to investigate this last point. Finally, in the model the particles are simplified to spheres while soot particles are complex fractal aggregates and the particle size is only defined by the diameter of the spheres. It can lead to inaccuracies but it has the merit to provide trends for the physical phenomenon investigated. 


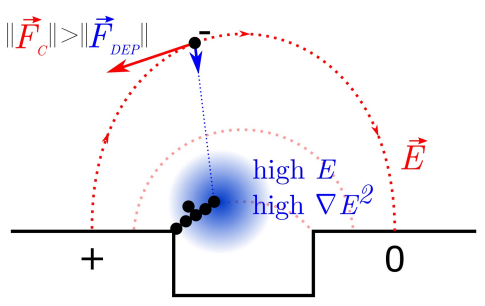

(a) Long range

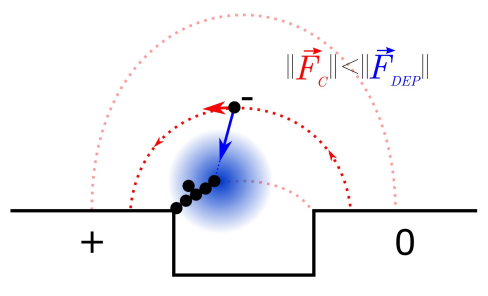

(b) Short range

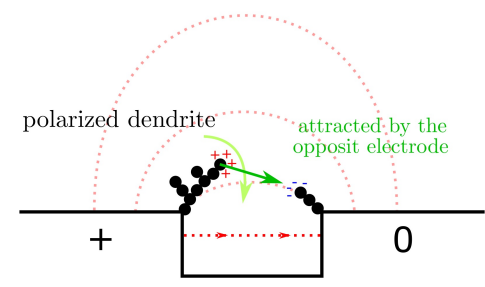

(c) Collapsing dendrites

Figure 14: Three possible mechanisms that may explain the dendrite growth on a resistive soot sensor. Mechanisms (a) and (b) are investigated in this work and mechanism (c) is an hypothesis to explain the collapse of the dendrites.

The hypothesis 2 (cf. Figure $14(\mathrm{c})$ ) is not investigated in this work because the model does not allow to numerically bend the dendrites. The work of Winter and Welland [38] provides an analytical model to compute the rotation of large non-spherical particles due to dielectrophoresis. They considered a prolate ellipsoid with a major axis length of $10 \mu \mathrm{m}$ in liquid (viscosity of $10^{-3} \mathrm{PI}$ ) and high field strength $\left(E>10^{6} \mathrm{~V} / \mathrm{m}\right)$. They concluded that the orientation time scale is of the order of $0.01 \mathrm{~s}$. This result is expected to be lower in the air because of the lower hydrodynamic torque. Thus, a similar phenomenon may also occur at the surface of the sensor but further investigations are required in order to quantify its relative magnitude.

\section{Conclusion}

The objective of this work was to investigate the physical phenomenon leading to the soot bridge-like structures building by means of numerical methods. To do so, a model inspired by the Teike et al. [19] model was implemented. The main feature of this model is the coupling between the particle deposition and the update of both the aerodynamic and electric field. The forces acting on particles include the brownian diffusion, the drag force and the Coulomb force. Thanks to this last force and for a given electric charge sign, the particles deposit on one side of the inter-electrode gap leading to a single dendrite.

The present study added one more term to the force balance: the dielectrophoresis. This choice is mainly justified by the geometry of the electrode studied in the present work. The inter-electrode gap is $20 \mu \mathrm{m}$ while it is $100 \mu \mathrm{m}$ wide in the Teike et al. study. The DEP force highly depends on the electric field gradient and it is problably neglectible in the Teike et al. case.

Nevertheless, it was found that the DEP force has a property that may explain the bridging of the electrodes by soot particles. The DEP does not depend on the particle electric charge sign. Particles are attracted both by grounded and polarized electrodes while they are necessarly repelled by one of them by the Coulomb force. Thus, one might not figure out how a bridge could "get closed" by the only action of the Coulomb force while the DEP explain this behavior.

Several simulations were carried out to understand how the DEP affects particle motion and dendrite growth. For all those simulations, particles carry the same electric charge and sign. First, the force magnitudes were compared for clean (with no dendrite growing) electrodes. At a short range, the DEP starts to be predominant for $300 \mathrm{~nm}$ spheres. The particle kinematic analysis shows that DEP attracts soot particles towards the corners of the electrodes because of the high electric field gradient intensity in this area, unlike the Coulomb force that attracts particle on the top surface electrode. Yet, the SEM micrograph shows that bridges are initiated on the angle of the electrodes. Finally, 2D dendrite growth was simulated and it was shown that a bridge may be achived for particles sensitive enough to the DEP force (500 $\mathrm{nm}$ diameter spheres). Moreover, even if the DEP is not intense enough to lead to a $100 \mathrm{~nm}$ diameter spheres bridge, it still has an impact on dendrite growth for small particles. 
Bridge-like structures were observed experimentally for electrostatically classified aerosols, that is to say for aerosols composed of particles within the same electric charge and sign. The SEM micrograph revealed a deposition pattern on the grounded and polarized electrode, suggesting the action of the Coulomb force at a long range. Furthermore, at the scale of the inter-electrode gap, bridges are observed which could be explained by the action of the DEP according to the theory and the numerical analysis performed in this work.

This model could be improved by implementing the bending process of the dendrites. They are considered stationnary but they could be rotated by the DEP force. Moreover, the implementation of a 3D model could improve the accuracy of the dendrite shape prediction. It is however expected to be computationnaly expensive because of the electric field and velocity flow updates. Another way to improve this model is to include a polydisperse approach and to have to ability to consider different charge distribution. Experiments with uncharged particles (using an electrostatic trap upstream of the sensor) is necessary to confirm the role of the DEP. Finally, more data about the actual shape of the bridges would be helpful to compare the simulated dendrites.

\section{References}

[1] B. Giechaskiel, A. Joshi, L. Ntziachristos, and P. Dilara. European regulatory framework and particulate matter emissions of gasoline light-duty vehicles: a review. Catalysts, 9(7):586, 2019.

[2] B. Giechaskiel, T. Lähde, and Y. Drossinos. Regulating particle number measurement from the tailpipe of light-duty vehicles: the next set? Environmental research, 172:1-9, 2019.

[3] A. Sassi, E. Rohart, and G. Belot. Post-traitement des émissions polluantes des moteurs thermiques à combustion interne - moteurs à allumage par compression. Techniques de l'ingénieur, 2011.

[4] R. W. Atkinson, H. R. Anderson, J. Sunyer, J. Ayres, M. Baccini, J. M. Vonk, A. Boumghar, F. Forastiere, B. Forsberg, G. Touloumi, J. Schwartz, and K. Katsouyanni. Acute effects of particulate air pollution on respiratory admissions: Results from aphea 2 project. American Journal of respiratory and critical care medicine, 164:1860-1866, 2001.

[5] B. Brunekreef and S. T. Holgate. Air pollution and health. The Lancet, 360(9341):1233-1242, 2002.

[6] J. Heyder. Deposition of inhaled particles in the human respiratory tract and consequences for regional targeting in respiratory drug delivery. Proc Am Thorac Soc, 1(4):315-320, 2004.

[7] G. Fischerauer, M. Förster, and R. Moos. Sensing the soot load in automotive diesel particulate filters by microwave methods. Measurement Science and Technology, 21(3):035108, 2010.

[8] L. Ntziachristos, P. Fragkiadoulakis, Z. Samaras, K. Janka, and J. Tikkanen. Exhaust particle sensor for obd application. In SAE 2011 World Congress \& Exhibition, SAE Technical Paper Series. SAE International400 Commonwealth Drive, Warrendale, PA, United States, 2011.

[9] M. M. Maricq, D. Bilby. The impact of the voltage and flow on the electrostatic soot sensor and the implications for its use as a diesel particulate monitor. Journal of Aerosol Science, 124:41-53, 2018.

[10] T. Kamimoto. A review of soot sensors considered for on-board diagnostics application. International Journal of Engine Research, 2016.

[11] A. Reynaud, M. Leblanc, S. Zinola, P. Breuil, and J.-P. Viricelle. Responses of a resistive soot sensor to different mono-disperse soot aerosols. Sensors (Basel, Switzerland), 19(3), 2019. 
[12] O. Brunel, F. Duault, J. Lavy, Y. Creff, and B. Youssef. Smart soot sensor for particulate filter obd. SAE International Journal of Passenger Cars - Electronic and Electrical Systems, 6(1):307$327,2013$.

[13] D. Grondin, A. Westermann, P. Breuil, J.-P. Viricelle, and P. Vernoux. Influence of key parameters on the response of a resistive soot sensor. Sensors and Actuators B: Chemical, 236:1036-1043, 2016.

[14] A. Malik, H. Abdulhamid, J. Pagels, J. Rissler, M. Lindskog, P. Nilsson, R. Bjorklund, P. Jozsa, J. Visser, A. Spetz, and M. Sanati. A potential soot mass determination method from resistivity measurement of thermophoretically deposited soot. Aerosol Science and Technology, 45(2):284$294,2011$.

[15] G. Hagen, C. Spannbauer, M. Feulner, J. Kita, A. Müller, and R. Moos. Conductometric soot sensors: Internally caused thermophoresis as an important undesired side effect. Sensors (Basel, Switzerland), 18(10), 2018.

[16] M. Feulner, G. Hagen, A. Muller, A. Schott, C. Zollner, D. Bruggemann, and R. Moos. Conductometric sensor for soot mass flow detection in exhausts of internal combustion engines. Sensors (Basel, Switzerland), 15(11):28796-28806, 2015.

[17] T. Ochs, H. Schittenhelm, A. Genssle, and B. Kamp. Particulate matter sensor for on board diagnostics (obd) of diesel particulate filters (dpf). SAE International Journal of Fuels and Lubricants, $3(1): 61-69,2010$.

[18] H. Husted, G. Roth, S. Nelson, L. Hocken, G. Fulks, and D. Racine. Sensing of particulate matter for on-board diagnosis of particulate filters. SAE International Journal of Engines, 5(2):235-247, 2012.

[19] G. Teike, M. Dietzel, B. Michaelis, H. Schomburg, and M. Sommerfeld. Multiscale latticeboltzmann approach for electrophoretic particle deposition. Aerosol Science and Technology, 46(4):451-464, 2012.

[20] P. Fragkiadoulakis, S. Geivanidis, and Z. Samaras. Modeling a resistive soot sensor by particle deposition mechanisms. Journal of Aerosol Science, 123:76-90, 2018.

[21] H. A. Pohl. Dielectrophoretic force. Journal of theoritical biology, 37:1-13, 1972.

[22] A. R. Minerick. Dc dielectrophoresis in lab-on-a-chip devices. In Dongqing Li, editor, Encyclopedia of Microfluidics and Nanofluidics, pages 1-9. Springer US, Boston, MA, 2013.

[23] F. J. Romay, Y. H. Liu, and S.-J. Chae. Experimental study of electrostatic capture mechanisms in commercial electret filters. Aerosol Science and Technology, 28:224-234, 1998. https://doi.org/10.1080/02786829808965523.

[24] M. Kerner, K. Schmidt, A. Hellmann, S. Schumacher, M. Pitz, C. Asbach, S. Ripperger, and S. Antonyuk. Numerical and experimental study of submicron aerosol deposition in electret microfiber nonwovens. Journal of Aerosol Science, 122:32-44, 2018.

[25] Z. Chen and X. Shen. Study on dielectrophoretic deposition of airborne particles in a vertical micro channel. Building and Environment, 45(4):968-975, 2010.

[26] H. S. Wasisto, S. Merzsch, A. Waag, E. Uhde, T. Salthammer, and E. Peiner. Airborne engineered nanoparticle mass sensor based on a silicon resonant cantilever. Sensors and Actuators B: Chemical, 180:77-89, 2013.

[27] Q. Zou and X. He. On pressure and velocity flow boundary conditions for the lattice boltzmann bgk model. 
[28] A. Reynaud. Ph. D., Compréhension et modélisation des mécanismes de captation des aérosols par couplage des phénomènes aérodynamiques et électriques. $\mathrm{PhD}$ thesis, Université de Lyon, Mines de Saint-Étienne, IFP Énergies Nouvelles, Saint-Etienne, 2019.

[29] D. Meeker. Finite elements method magnetics website, 2019.

[30] L. Verlet. Computer experiments on classical fluids. i. thermodynamical properties of lennardjones molecules. Physical Review, 159(1):98-103, 1967.

[31] J. W. Lee S., S. H. Lee, and J. Jang. Numerical analysis on the electrostatic capture of airborne nanoparticles and viruses in a homemade particle concentrator without a unipolar charger. Journal of Electrostatics, 70(2):192-200, 2012.

[32] D. Gupta and M. H. Peters. A Brownian dynamics simulation of aerosol deposition onto spherical collectors. Journal of Colloid and Interface Science, 104(2):375-389, April 1985.

[33] M. D. Allen and O. G. Raabe. Slip correction measurements of spherical solid aerosol particles in an improved millikan apparatus. Aerosol Science and Technology, 4:269-286, 1985.

[34] W. C. Hinds. Aerosol technology: Properties, behaviour, and measurement of airborne particles. Wiley, New York and Chichester, 2nd ed. edition, 1999.

[35] N. A. Fuchs. The Mechanics of Aerosols. Pergamon, New York, 1964.

[36] D. B. Kittelson, D. Y. H. Pui, and K. C. Moon. Electrostatic collection of diesel particles. SAE Technical Paper, 1986.

[37] D. Grondin. Ph. D., Développement d'un capteur de suie pour application automobile: Etude des paramètres clés affectant sa réponse. PhD thesis, Ecole des Mines de Saint-Etienne, Saint-Etienne, 2017.

[38] W. T. Winter and M. E. Welland. Dielectrophoresis of non-spherical particles. Journal of Physics D: Applied Physics, 42(4):045501, 2009. 